\title{
Antimicrobial Resistance Genes and Bacteria Detected in Hospital Sewage May Provide Valuable Information in Clinical Antimicrobial Resistance
}

\section{Leshan Cai}

The First Affiliated Hospital of Shantou University Medical College

Jiayu Sun

Shantou University Medical College

Fen Yao

Shantou University Medical College

Qiaoxin Zhang

The First Affiliated Hospital of Shantou University Medical College

\section{Yumeng Yuan}

Shantou University Medical College

\section{Qindong Xie}

Shantou University Medical College

\section{Shiwei Wang}

Yangzhou University Medical Academy

\section{Zhen Wang}

Shantou University

Xiaoyan Jiao ( $\nabla$ xyjiao@stu.edu.cn )

Shantou University Medical College https://orcid.org/0000-0002-7568-0595

\section{Research}

Keywords: Antibiotics, Antimicrobial-resistant bacteria, Antimicrobial-resistant gene, Sewage, Metagenomics

Posted Date: October 26th, 2020

DOI: https://doi.org/10.21203/rs.3.rs-93424/v1

License: (1) (1) This work is licensed under a Creative Commons Attribution 4.0 International License.

Read Full License 


\section{Abstract}

Aim: Clinical antimicrobial resistance (AMR) is a significant threat to public health, which is often unclear due to representative data from human populations that are challenging to obtain. Study the associations between consumption of antibiotics and antimicrobial resistance bacteria or genes (ARB or ARGs) that will benefit from elucidating the AMR.

Methods: The details of antibiotics usage were calculated based on the actual consumption in the target hospital, ARB was detected by culture method, and ARGs were evaluated by metagenomics.

Results: Our study revealed that culture-based single-indicator-strain approaches only capture the AMR in $16.17 \%$ infectious samples. 1573 bacterial species and 885 types of ARGs were found in the hospital sewage. The consumption of antibiotics influences the resistance profiles that were significant in E.coli, but the strength varies among bacteria. In all ARGs group, ARGs of aminoglycosides was the most common, followed by sulfonamide, tetracycline, phenicol, macrolides, and quinolones, comprising to $82.6 \%$ of all ARGs. Five hundred nineteen pairs of ARGs and bacterial species showed a significant correlation ( $r>0.8)$. The co-occurrence patterns of bacteria- ARGs mirrors the AMR of the clinic. Antibiotic usage will affect the abundance of ARG in sewage, with a hysteresis effect.

Conclusion: The ARGs- bacteria co-occurrence patterns from wastewater could be a valuable bio-indicator to reflect the emergence of ARB in the future. Developing a predictive risk model of AMR on this basis will facilitate the rational use of antibiotics.

\section{Introduction}

Antimicrobial resistance (AMR) is a global health crisis. Antibiotics are extensively used for empirical treatment and prophylactically, inducing the propagation of antibiotic-resistant bacteria (ARB) and genes (ARGs). In the clinic, AMR affects not only the therapeutic efficacy of patients with primary infection but also the increasing number of hospital-acquired infections predispose patients to secondary disease, i.e., nosocomial infections during hospitalization. Significantly increasing AMR led to infections that were once easily treatable but now hardly control. Reliable information that accurately describes and characterizes the occurrence and transmission of AMR is a benefit to treatment decisions. However, obtaining representative data on AMR for healthy human populations is difficult as AMR is a complex problem with multiple and interconnected drivers [1].

In hospitals, AMRs are mainly evaluated by culture-based methods. Unfortunately, the vast majority of microbes cannot grow outside their host environment [2]. The difficulty of collecting specimens, especially infection occurring in deep organs of the body, plus the long time for bacterial isolation, clinicians can hardly get a timely report about AMR to guide their treatment choice. That prescription could not base on antibiotic sensitivity results in aggravate AMR. More important, the culture-based single-indicator-strain approaches cannot capture the AMR and ARGs in real microbial communities, that severely hinders exploration of the mechanisms behind drug resistance. qPCR method can identify the 
unculturable bacteria and ARGs but is not readily available for the discovery of unknown ARBs or nonexpressed ARGs[3]. Current surveillance of AMR is often focusing on specific pathogens isolated from clinical infectious samples, this procedure leads to significant time delays, often incomparable data, and a narrow pathogen spectrum not capturing all relevant AMR genes $[1,4,5]$. General information regarding AMR in the hospital environment has more clinical significance than the data from a single patient or an antibiotic sensitivity test, an appropriate method reflecting hospital AMR status is of considerable importance to promote antibiotic therapeutic efficacy.

Hospitals are unique places with extensive usage of antibiotic drugs on patients. A high average dose prescription of antibiotics for inpatient users may result in higher concentrations of ARB. Then, antibiotic prescription trends in local hospitals may play a critical role in ARB distribution in the environment. Besides ARB, medical waste, including the routine application of antimicrobial chemicals for sanitation, and antibiotics excreted in hospital wastewater, making it a reservoir of ARB and ARGs. Different types of antibiotics presenting in wastewater that may play a role in the selection of resistance would occur and contribute to resistance retention and dissemination[6], which may promote ARG occurrence [7]. High densities of ARB could facilitate the propagation and spread of ARGs by horizontal gene transfer via plasmids, transposons, and integrons [8, 9]. Most importantly, ARB and ARGs in hospital sewage directly come from clinical practice, and it does not contain the contamination from antibiotic sources from industry and agriculture. Therefore, hospital sewage may be the most appropriate specimen to study the associations among antibiotics use, AMR, and ARGs.

Nowadays, metagenomics is becoming a potential tool in the discovery of environmental ARGs, resulting in a paradigm shift from phenotype- to genotype-based diagnostics of AMR. Currently, there is little data revealed the association among antibiotics, ARB, and ARGs in the hospital settings. In this study, overall antibiotic use in hospital, clinical AMR information was from clinical isolate, as well as the ARGs profiles in hospital sewage were collected. The correlations among them was analyzed. Our results may provide valuable information on the effect of antibiotic use on clinical AMR.

\section{Methods}

The study was approved by the Shantou University Medical College Institutional Review Board. A small urban general hospital with 250 beds was selected as the target hospital. Sewage samples were collected from an urban hospital wastewater outlet (Shantou, China). $1 \mathrm{~L}$ sewage sample was collected weekly in sterile bottles and transported on ice to the diagnostic microbiology laboratory within $4 \mathrm{~h}$ of collection from June 2018 to November 2018. In total, 30 samples were collected, and each sample was then centrifuged at $10000 \mathrm{rpm}$ for 5 minutes, and sediments were stored at $-80^{\circ} \mathrm{C}$ before the experiment.

\subsection{Antibiotics use}

To investigate the effect of antibiotic administration on the abundance and variety of human bacterial pathogen (HBPs), we collected the details of antibiotic usage in the whole year of the target hospital. All 
types of antibiotics were classified into eight categories.

\subsection{HBPs identification and antimicrobial susceptibility testing}

Bacteria isolated from hospitalized patients' infectious samples were identified using an automated ATB microbial identification system (bioMérieux, Marcy-l'Etoile, France) according to the standard protocol of Shantou University Medical College (see Appendix A for more details). ATB G-CLSI (12) kit was used for Gram-negative bacilli testing, while the ATB STAPH CLSI (12) kit was used for Gram-positive cocci (Staphylococcus) drug susceptibility testing. A $24 \mathrm{~h}$ pure culture colony was prepared for drug sensitivity results. The Minimum Inhibitory Concentration (MIC)for common antibiotics were determined for all isolates, and antimicrobial susceptibility testing was then conducted for 26 antimicrobials (see Supplement Table 1-2 for more details). The drug susceptibility test of bacterial isolated seen in supplementary materials.

\subsection{MGS sequencing}

\subsubsection{DNA extraction and qualification:}

Mixed sediments (monthly) were thawed at room temperature $\left(22^{\circ} \mathrm{C}\right)$, and microbial DNA was extracted using the E.Z.N.A. ${ }^{\circledR}$ soil DNA Kit (Omega Bio-Tek, Norcross, GA, U.S.) according to manufacturer's protocols. The DNA concentration was measured using Qubit ${ }^{\circledR}$ dsDNA Assay KitinQubit ${ }^{\circledR} 2.0$ Fluorometer (Life Technologies, CA, USA). OD value is between 1.8-2.0. DNA contents above $1 \mu \mathrm{g}$ were used to construct the library. Sequencing libraries were generated using NEB Next ${ }^{\circledR} U$ Ultra $^{\text {TM }}$ DNA Library Prep Kit for Illumina (NEB, USA). PCR products were purified (AMPure XP system) and libraries were analyzed for size distribution by Agilent2100 Bioanalyzer and quantified using PCR. The clustering of the index-coded samples was performed on a cBot Cluster Generation System. After cluster generation, the library preparations were sequenced on an Illumina platform, and paired-end reads were generated.

\subsubsection{Network and statistics analysis}

To explore the underlying associations within/among bacteria and ARGs, we constructed a correlation matrix by calculating all pairwise Spearman correlation coefficients $(r)$ among bacterial species and ARG subtypes that occurred in at least $50 \%$ of all samples. A correlation between ARG-ARG, or species-species, or ARG-species was considered as statistically significant and robust if the adjusted P-value (by multiple testing corrections via false discovery rate (FDR) estimation) was $<=0.01$, and the Spearman 's $r$ was $>$ 0.8 . All the robust correlations identified from pairwise comparison of abundance form a correlation network[10].

\subsection{Statistical analyses}

ANOVA, Kruskal Wallis, LEfSe, and DEseq2 were employed to identify the bacteria with different abundance among samples and groups [11]. Co-occurrence analysis was performed by Spearman's rank 
correlations between predominant taxa, and the network plot was used to display the associations among taxa. Unless specified above, parameters used in the analysis were set as default. The reads were de-novo assembled using Megahit assembler, and the quality of assembly was examined by Quast [12]. Prodigal was used to predict the potential functional genes in assembled contigs [13]. The reads were taxonomically classified using Kraken2 [14]. Kraken2 matches each K-mer within a query sequence to the lowest common ancestor (LCA) of all genomes in the database containing the given K-mer.

The models calculated the changes of ARGs associated with one kind of antibiotic consumption per monthly dose, appropriate estimates of the antibiotic selection pressure throughout treatment. Besides, we utilized a correlation-based network to analyze the non-random ARG-ARG, and ARG-HBP cooccurrence patterns in the sewage sludge, which helped to propose a novel approach for risk assessment of ARGs acquisition by HBPs in human-impacted environments.

\section{Results}

\subsection{Antibiotic consumption in target hospital}

The annual consumption of antibiotics and their temporal distribution was calculated in the target hospital, based on the total amount of antibiotics issued by hospital pharmacies. The dosage of used antibiotics was plotted in terms of months. Among all antibiotics, the most used antibiotic was Cephalosporin, its consumption fluctuates in the range of $2000-4000 \mathrm{~g} / \mathrm{month}$ throughout the year. The second antibiotics were $\beta$-lactase inhibitors, which remained at $1000-2000 \mathrm{~g} / \mathrm{month}$ from January to April, the usage continued to decrease to as low as $200 \mathrm{~g} / \mathrm{month}$ from May to August, and then gradually elevated to about $1000 \mathrm{~g} /$ month after August. The third antibiotics were Quinolones; the consumption varies throughout the year. Penicillin usage was more than Nitroimidazole from January to March, but the dosage of Nitroimidazole continued to rise from April, more than the amount of penicillin. The consumption of Aminoglycosides, Carbapenem, and Macrolides kept below $80 \mathrm{~g} / \mathrm{month}$, and their monthly dosage fluctuates slightly. The annual curve of antibiotic use showed in Fig. 1

\subsection{Bacteria identifications from clinical samples and its antibiotic resistance profiles}

From January to December 2018, a total of 643 samples were collected from blood, urine, sputum, the stool of infectious patients. Bacteria were isolated from $104(16.17 \%)$ samples by culture, while 539 (83.83\%) samples were culture negative. The most common isolates were Escherichia coli (E. coli), following Klebsiella pneumonia, Staphylococcus aureus, Pseudomonas aeruginosa, and Proteus mirabilis, which comprised up to $70 \%$ of all isolates.

Antibiotic resistance profiles were obtained for all bacteria isolates. ARBs were classified into eight groups according to the type of antibiotic, including penicillin, $\beta$-lactamase inhibitor, Cephalosporins, Carbapenem, Quinolones, Macrolides, Aminoglycosides, and Nitroimidazole (Table 1). Cephalosporin, $\beta$ lactamase inhibitor, and Quinolones were the top three types of antibiotics used in the hospital, while Macrolides were the minimal use antibiotic. Our results suggested that the effect of antibiotic use on ARB 
was significant. Similar trends were also found in K. pneumoniae and P. mirabilis. S. aureus was found to have high resistance to $\beta$-lactamase (91.6\%), middle resistance to Tetracycline (33.3\%), Lincosamides (41.7\%), low resistance to Quinolones (25\%), Aminoglycosides (25\%), Macrolides (25\%), and Chloramphenicol (25\%). In particular, the rate of resistance to $\beta$-lactamase was the highest in $P$. aeruginosa. Resistance to at least three antimicrobials was found at a high frequency in clinical isolates as follows: E. coli (72.2\%), P. mirabilis (66.7\%), P. aeruginosa (66.7\%), S. aureus (41.7\%), and $K$. pneumoniae (26.7\%) (Table 1). Our results indicated that minimal bacteria could be detected by culture method, and the multi-drug resistance was very high among the isolated bacteria, suggesting that clinical AMR was severe in this hospital. 
Table 1

Bacteria isolated from the clinic and their resistance profiles

\begin{tabular}{|c|c|c|c|c|c|}
\hline Bacteria & Antibiotic Type & $\begin{array}{l}\text { Drug resistance } \\
\text { rate }(\%)\end{array}$ & Bacteria & Antibiotic Type & $\begin{array}{l}\text { Drug } \\
\text { resistance } \\
\text { rate(\%) }\end{array}$ \\
\hline \multirow[t]{8}{*}{ E. coli } & Carbapenems & 0 & \multirow{8}{*}{$\begin{array}{l}\text { K. } \\
\text { pneumoniae }\end{array}$} & Carbapenems & 0 \\
\hline & Penicillin & 91.7 & & Penicillin & 86.7 \\
\hline & $\begin{array}{l}\beta \text {-lactamase } \\
\text { inhibitor }\end{array}$ & 13.9 & & $\begin{array}{l}\beta \text {-lactamase } \\
\text { inhibitor }\end{array}$ & 20.0 \\
\hline & Cephalosporins & 83.8 & & Cephalosporins & 33.3 \\
\hline & Aminoglycosides & 47.2 & & Aminoglycosides & 26.7 \\
\hline & Quinolones & 88.9 & & Quinolones & 33.3 \\
\hline & Tetracyclines & 77.8 & & Tetracyclines & 40.0 \\
\hline & $\begin{array}{l}\text { Multi-drug } \\
\text { resistance }\end{array}$ & 72.2 & & $\begin{array}{l}\text { Multi-drug } \\
\text { resistance }\end{array}$ & 26.7 \\
\hline \multirow{8}{*}{$\begin{array}{l}P . \\
\text { aeruginosa }\end{array}$} & Carbapenems & 50 & \multirow[t]{8}{*}{ P. mirabilis } & Carbapenems & 0 \\
\hline & Penicillins & 66.7 & & Penicillins & 66.7 \\
\hline & $\begin{array}{l}\beta \text {-lactamase } \\
\text { inhibitor }\end{array}$ & 100 & & $\begin{array}{l}\beta \text {-lactamase } \\
\text { inhibitor }\end{array}$ & 0.0 \\
\hline & Cephalosporins & 50.0 & & Cephalosporins & 66.7 \\
\hline & Aminoglycosides & 66.7 & & Aminoglycosides & 50.0 \\
\hline & Quinolones & 50.0 & & Quinolones & 50.0 \\
\hline & Tetracyclines & 16.7 & & Tetracyclines & 100.0 \\
\hline & $\begin{array}{l}\text { Multi-drug } \\
\text { resistance }\end{array}$ & 66.7 & & $\begin{array}{l}\text { Multi-drug } \\
\text { resistance }\end{array}$ & 66.7 \\
\hline \multirow[t]{7}{*}{ S. aureus } & Penicillins & 91.6 & & & \\
\hline & Aminoglycoside & 25.0 & & & \\
\hline & Macrolides & 25.0 & & & \\
\hline & Lincosamide & 41.7 & & & \\
\hline & Tetracyclines & 33.3 & & & \\
\hline & Quinolones & 25.0 & & & \\
\hline & $\begin{array}{l}\text { Multi-drug } \\
\text { resistance }\end{array}$ & 41.7 & & & \\
\hline
\end{tabular}




\subsection{Diversity, richness, and composition of microbial communities in hospital sewage}

Altogether, 21 bacterial phyla were identified from sewage samples. The dominant phyla were proteobacteria (82.19\%), followed by Bacteroides (7.94\%), actinobacteria (5.90\%), and firmicutes $2.95 \%)$. Specifically, 46 classes, 89 orders, 191 families, 629 genera, and 1573 species were found. The top five bacterial species were E.coli (7.60\%), Hydrogenophaga_pseudoflava (5.42\%), Reyranella_massiliensis (3.68\%), Klebsiella_pneumoniae (3.43\%), and Acidovorax sp. KKS102 (3.02\%). Among them, E.coli and Klebsiella_pneumoniae were the most bacteria isolated from patients. The sequences described in this study were deposited in NCBI Sequence Read Archive with accession numbers (PRJNA612238).

\subsection{Occurrence and abundance of ARG types in hospital sewage}

In total, 885 types of ARGs were detected in hospital sewage. All ARGs were mapped to their corresponding antibiotics that were frequently used in the clinic. If an ARG was found to confer resistance to more than two types of antibiotics, it was categorized as "multi-drug" resistance. Hence, we subgrouped ARGs into the Aminocoumarin, Aminoglycosides, Diaminopyrimidine, Dual-resistance genes, Macrolides, Nitroimidazole, Penicillin, Peptide, Phenicol, Quinolones, Rifamycin, Tetracycline, Sulfonamide, and multiple resistance genes. ARGs conferring resistance to Aminoglycosides accounted for $24.57 \%$, which was the most abundant in all ARGs group, followed by Sulfonamide (15.7\%), Tetracycline (10.51\%), Phenicol (7.92\%), Macrolides (5.03\%), Quinolones (3.96\%). Those groups contributed $82.56 \%$ of all ARGs. For an single ARG, the most abundant ARG were sul1 (10.33\%), followed by APH(6)-ld (5.86\%), APH(3")-lb (5.56\%), Nocardia (5.54\%), sul2 (5.14\%), tet (G) (5.05\%), ErmF (4.39\%), pp-flo (3.44\%), AAC(6')-lb7(3.27\%), floR (2.83\%), tet (C) (2.14\%), tetX (2.0\%). The abundance between 1-

$2 \%$ were ugd, emrA, OXA-45, EreA, msrE, mtrA, mphD, ANT(2")-la, qacH, and AAC(6')-lb8. The rest accounted for $<1 \%$. The types of resistance genes detected in the sewage could match the resistance profile in our hospital. The ARG profiles were compared using Bray-Curtis based non-metric multidimensional scaling (NMDS) plots in $\mathrm{R}$ with the vegan package [15]. Heatmaps were used to display the abundances of individual ARGs (FIG.2).

\subsection{The effect of antibiotic consumption on the distribution of ARB and ARGs}

Our study revealed that antibiotics usage influenced the distribution of ARB. The most obvious example was observed in E. coli. Antibiotic prescription in target hospital reported that a higher average monthly dose prescription of Cephalosporin, $\beta$-lactamase inhibitor, Quinolones and Penicillin for their inpatient users, and higher ratios of $E$. coli resistant to Cephalosporin and $\beta$-lactamase inhibitor was found in clinical samples. The curve of the antibiotics usage was very close to the curve of the isolation rate of 
ARB. P. aeruginosa had high resistance to Cephalosporin, and $P$. mirabilis had high strength to Quinolones.

The effects of antibiotic use on ARGs were analyzed by studying their time distributions. Our results revealed that antibiotic usage would affect the abundance of ARG in sewage, with a hysteresis effect. For aminoglycosides, its consumption increased between February and April, decreased from April to May, and increased again. Accordingly, ARGs of aminoglycosides grew from June to August, with a time delay of about four months, both of which had very similar curves over time. The phenomenon was also observed in ARGs to $\beta$-lactamase inhibitor, macrolides, and penicillin. The changes in ARGs abundance were delayed by 2 to 6 months compared with changes in antibiotic dosage. Our results revealed that antibiotics consumption could cause an alteration in the compositions and abundances of dominant ARB and ARG types(FIG.3)

\subsection{Co-occurrence pattern of ARB and ARGs}

A network approach was used to explore the non-random correlations between ARG-ARG, ARG-bacterial species, and species -species in hospital sewage. Altogether, the network contribution mode was 6903 pairs, in which the random mode of correlations was 6384 pairs, while the non-random mode was 519 pairs. The significant and robust correlations were observed in 10 species-species, 147 ARG-species, and 362 ARG-ARG co-occurrences pattern $(r>0.8, P<0.05)$. Scale-free networks contain hubs with connectivity distribution between nodes based on power-law distribution. Most nodes have a few connections, but some of them, known as hubs, have many connections. Co-occurrence patterns were evident within or across ARG types, among them, PatA, CRP, and AAC(6')-30/AAC (6')-Ib' had the most cooccurring connections, while the $\operatorname{AAC}\left(6^{\prime}\right)-30 / A A C\left(6^{\prime}\right)-1 b^{\prime}$, baeR, emrB, aadA3, aadA25, cpxA, tet.G, and the multidrug resistance genes including $\mathrm{MexB}, \mathrm{smeE}$, acrB were located in the hub. The significant cooccurrence pattern also observed in ARGs and bacteria, including Rhodobacteraceae with the AAC.6...Ib8, OXA.45, and tet.G; Nocardia with bacA, mdtB, and ugd; Comamonadaceae with bacA, catB3, tet.G., and ugd; Campylobacteraceae with bacA, catB3, mdtB, tet.G, and ugd; Moraxellaceae with AAC.6...Ib8, and tet.G; Microbacteriaceae with aadA16, ANT.2....la, EreA, mtrA, tet.C., and tetX; Enterobacteriaceae with bacA, catB3, tet.G, and ugd; Bacteroidaceae with catB3, OXA.45, and ugd.

ARG subtypes were grouped into a broad category according to the types of antibiotics, and bacteria were selected from the clinical isolates and sewage. E.coli and K. pneumoniae either listed in the most isolates from hospitals or the most abundant bacteria found in wastewater. The network representing the correlation between ARG and bacteria contained 30 nodes, including 12 ARGs subtypes, 18 microbial taxa, and 36 edges. All connection stands for a significant relationship (Spearman's $r>0.8$ ). Significant correlations were observed in bacteria and ARGs, which partly overlap the clinical drug resistance phenotype (Fig. 4-5)

\section{Discussion}


The increasing AMR caused by the overuse of antibiotics has emerged as a significant threat to global health. A precise and timely report of $A R B$ is critical for reducing empirical antibiotic prescription, which is vital for reducing clinical AMR. Unfortunately, the unculturable or unknown isolation conditions make about $90 \%$ of bacteria that could not be cultured in vitro $[16,17]$. In our study, bacteria could be isolated in only $16.17 \%$ of infectious samples, AMR information obtained from isolated bacteria could not meet the requirement that can guide antibiotic use. Compared with the species isolated by culture, metagenomics had significantly higher effectiveness in detecting bacterial species, especially those in the environment. Up to 1573 bacterial species were found in the hospital sewage. Proteobacteria and Bacteroidetes were the main phyla identified, comprising a variety of gram-negative bacteria such as Escherichia, Salmonella, Vibrio, and other pathogenic genera. Among the high abundance bacterial species found in sewage, $E$. coli, K. pneumonia, P. aeruginosa, Acidovorax_sp._KKS102, P. mirabilis, and S. aureus were also the main bacteria isolated in hospital, which are eventually discharged into sewage. Thus, bacterial species in wastewater can reflect the total number of clinical bacteria.

The relationships between antibiotic use and AMR involve the hospital population, pathogen genotypes, intensity of infection control, and transmission dynamics [18-20]. When analyzing the effect of antibiotic consumption on AMR, antibiotic surveillance based on sales data describes an incomplete story. Building a nationwide surveillance program to quantify antibiotic prescriptions from all sources, i.e., pharmacies, clinics, hospitals, is quite challenging to achieve [21]. In our study, the dosage of antibiotics was calculated based on the actual consumption of hospitalized patients. Cephalosporin, $\beta$-lactase inhibitors, and Fluoroquinolones were the most frequently used antibiotics. Their high average monthly dose prescription results in higher levels of ARBs, including $E$. coli, $P$. aeruginosa, and $P$. mirabilis resistant to the corresponding antibiotic. The amount of antibiotics affects the distribution of ARBs with or without delay effect. A greater abundance of Proteobacteria identified in the hospital environment may be the result of antimicrobial use in the hospital $[7,22]$. Consistent with the previous study, our results also indicated the close correlations of the quantities of ARBs with those of corresponding antibiotics [23]. The reason for the correlation failed to find in low abundance bacteria, may due to the number of cases is too small to meet the statistical sample requirements. The difficulty in obtaining the samples from the vast population, the complexity of pathogens, behavioral and antibiotic response patterns, made antibiotic use guiding by bacterial sensitivity tests in a hospital almost impossible in the present period. In monitoring the occurrence of AMR, a significant advantage of hospital sewage is that such samples can be easily obtained and analyzed without ethical concerns compared to samples directly collected from humans [1].

The induction and development of ARGs could be related to the concentration effects of antibiotics on the bacterial groups harboring these genes [24]. For tracking the potential hosts of ARGs, the cooccurrence patterns between ARG subtypes and microbial taxa were explored by correlation analysis [25, 26]. In our study, more than 40 mobile ARGs were all from Proteobacteria, and many novel pairwise of ARG-bacteria or ARG-ARG examples observed from the network. E-coli, the most abundant bacterium isolated from our clinical samples, was highly resistant to Quinolones, Penicillin, Beta-lactamase inhibitor, Cephalosporin, Tetracycline, and Aminoglycosides. In the network, E-coli was co-occurrence with multiple 
ARGs, including AAC(6')-30/ AAC(6')Ib', acrD, acrF, baeR, CRP, cmlA5, emrA, marA, patA, pmrF, floR, and pp.flo, indicating that multi-resistance of E-coli may be induced by these ARGs. The intrinsic ARGs are merely antibiotic determinants conferring resistance phenotype. Thus, understanding the intrinsic resistome will, therefore, contribute to the prediction of the emergence and evolution of antibiotic resistance in the future $[27,28]$.

The human activities using antibiotics lead to significant amplification of the original ARGs in social clinical settings [29]. Underexposure to antibiotics, higher incidences of co-occurrence ARG subtypes of the same type may generally reflect selective pressure exerted by the same antibiotics in sewage, as our results showed, antibiotic consumption of a few months before influenced the abundance of ARGs in sewage later, with the peak of ARGs lagging that of antibiotics use for 2-6 months. Previous studies showed that Cephalosporin consumption of 7-10 months before had a significant influence on resistance, while consumption of Penicillin / Beta-lactamase inhibitor produced resistance without delay [30]. The possible explanation for this phenomenon may be that different antibiotics have different selective capabilities [31], and the abundance of ARGs is driven by complex interplays of different environmental, antibiotic types, and anthropogenic variables, rather than being related to any single factor. That clinical antibiotic usage affects the abundance and distribution of ARGs in sewage was marked in Aminoglycosides, $\beta$-lactamase inhibitor, Macrolides, Penicillin, and the enriched occurrence of these ARGs was closely associated with the antibiotic's usage in the target hospital.

The correlation between antibiotics and ARGs was not observed in tetracycline and the sulfonamide. A previous study revealed that ARGs of tetracycline and sulfonamide were the top three widely transferred ARGs [29]. Despite deficient consumption at present, a high abundance of ARGs to tetracycline were account for $10.49 \%$ of total ARGs. Consistent with the results of ARGs, high resistance to tetracycline was found in $80 \%$ E.coli, in $100 \%$ P. mirabilis, and in $75 \%$ K. pneumonia of clinical isolates. A possible explanation may be a potential source of ARGs in the environment was caused by the continuous prophylactic use of antibiotics as a for animals [32]. Another reason is that antibiotics use and their corresponding ARGs do not always appear simultaneously [33].

The inter-types co-occurrence of ARGs, such as beta-lactam and aminoglycoside resistance by methicillin resistant $S$. aureus [34] and prevalent co-resistance to Aminoglycosides, Tetracycline, and Quinolones by beta-lactamase (ESBL) producers [35], are more likely driven by a co-resistance mechanism, i.e., presence of different resistance determinants on the same genetic element [36]. Our correlation network revealed bacteria bearing multiple resistance genes, as well as cross-resistance between antibiotics. The most abundance ARG, sul1, usually found in the conserved region of class 1 integrons, is capable of transferring between bacteria in different environments. Other high abundance ARGs, like the AAC.6...Ib8, $\mathrm{AAC}\left(6^{\prime}\right)-30 / \mathrm{AAC}\left(6^{\prime}\right) \mathrm{lb}$ ', patA, mexF, aadA3, and QnrS2, located in the hub of a network, may own high potential risks for horizontal gene transfer between different bacteria in the sewage. ARGs, including aadA1, aac(3)-II and aph(6)-I ( aminoglycoside ), tetA and tetG (tetracycline ), sul1 and sul2 (sulfonamide ), catA and catB (chloramphenicol), were carried by the same genome [37]. When the genes that cause resistance phenotypes are located together on the same genetic element (MGEs), such as a plasmid, 
transposon, or integron, co-resistance occurs $[38,39]$. This information may provide some clues regarding multi-drug resistance ARBs happened. The correlations between ARGs and bacterial taxa in the network could provide some insights into the potential mechanism of human pathogens prone to the acquisition of ARGs [40]. Based on the ARGs located in the hub of a network, and we could track the possible routes of ARGs transmission and dissemination among ARB. The interactions between the number of resistant bacteria and antibiotics may have contributed to a significant increase of some ARGs, which confer resistance to the most frequently used antibiotics in the hospitals [41, 42]. Therefore, the co-occurrence patterns between bacteria and the ARGs could help to propose a novel approach for risk assessment of ARGs acquisition by HBPs in human-impacted environments [10], and the future level of resistance, esp. short- to medium-term, could be forecasted based on antibiotic usage and incidence of bacteria [30], though it remains unclear for the diversity, distribution, and fate of ARGs in urban water systems [43].

Hospital discharges are essential reservoirs of ARB and ARGs [44, 45], which promote the potential spread of AMR to the environment [46, 47]. More attention should be paid to assess ARGs in hospital sewage, which could provide a great solution to clinical AMR monitoring.

\section{Conclusion}

The ARGs- bacteria co-occurrence patterns from wastewater could be a valuable bio-indicator to reflect the emergence of ARB in the future. Developing a predictive risk model of AMR on this basis will facilitate the rational use of antibiotics.

\section{Abbreviations}

AMR

antimicrobial resistance, ARB:antimicrobial-resistant bacteria, ARGs:antimicrobial-resistant genes, HBPs:Human bacterial pathogens

\section{Declarations}

\section{Availability of data and materials}

The datasets used and analyzed in the context of this survey are available from corresponding author upon reasonable request.

\section{Competing interests}

All authors declare that they have no competing interests

\section{Acknowledgments}

We would like to thank Dr. Minjie Luo from Shantou University Medical College for providing scientific advice and a critical review of the study for English language editing 


\section{Authors' Contributions}

Leshan Cai and Jiayu Sun carried out the Sample collection, experimental studies and drafted the manuscript. Fen Yao and Qiaoxin Zhang participated in the design of the study. Yumeng Yuang, Zhen Wang, Shiwei Wang,Qindong Xie participating the experiments and performed the statistical analysis. Xiaoyang Jiao conceived of the study, participated in its design and coordination and helped to revise the manuscript. All authors read and approved the final manuscript

\section{Funding}

This study was supported by the Shantou Science and Technology Project (grant numbers: 180709174010328), Guangdong Medical Research Project (grant numbers: B2019181), and 2020 Li Ka Shing Foundation Cross-Disciplinary Research Grant (Project Number: 2020LKSFG03E) for collection, validation and analysis of data.

\section{Ethics approval and consent to participate}

The study was approved by the Ethics Committee of the First Affiliated Hospital of Shantou University Medical College. Since it does not involve human specimens, it does not need to be approved by the Ethics Committee.

\section{Consent for publication}

Not applicable

\section{References}

1. Hendriksen RS, Munk P, Njage P, van Bunnik B, McNally L, Lukjancenko O, Roder T, Nieuwenhuijse D, Pedersen SK, Kjeldgaard J, Kaas RS, Clausen P, Vogt JK, Leekitcharoenphon P, van de Schans MGM, Zuidema T, de Roda Husman AM, Rasmussen S, B Petersen, c. Global Sewage Surveillance project, C Amid, Cochrane G, Sicheritz-Ponten T, Schmitt H, Alvarez JRM, Aidara-Kane A, Pamp SJ, Lund O, Hald T, Woolhouse M, Koopmans MP, Vigre H, Petersen TN, Aarestrup FM, Global monitoring of antimicrobial resistance based on metagenomics analyses of urban sewage, Nat Commun, 10 (2019) 1124.

2. Schmieder R, Lim YW, Edwards R. Identification and removal of ribosomal RNA sequences from metatranscriptomes. Bioinformatics. 2012;28:433-5.

3. Bondarczuk K, Markowicz A, Piotrowska-Seget Z. The urgent need for risk assessment on the antibiotic resistance spread via sewage sludge land application. Environ Int. 2016;87:49-55.

4. Hay SI, Rao PC, Dolecek C, Day NPJ, Stergachis A, Lopez AD, Murray CJL. Measuring and mapping the global burden of antimicrobial resistance. BMC Med. 2018;16:78.

5. Zignol M, Dean AS, Falzon D, van Gemert W, Wright A, van Deun A, Portaels F, Laszlo A, Espinal MA, Pablos-Mendez A, Bloom A, Aziz MA, Weyer K, Jaramillo E, Nunn P, Floyd K, Raviglione MC. Twenty 
Years of Global Surveillance of Antituberculosis-Drug Resistance. N Engl J Med. 2016;375:1081-9.

6. Aminov RI, Mackie RI. Evolution and ecology of antibiotic resistance genes. FEMS Microbiol Lett. 2007;271:147-61.

7. He P, Wu Y, Huang W, Wu X, Lv J, Liu P, Bu L, Bai Z, Chen S, Feng W, Yang Z. Characteristics of and variation in airborne ARGs among urban hospitals and adjacent urban and suburban communities: $A$ metagenomic approach. Environ Int. 2020;139:105625.

8. Stalder T, Barraud O, Jove T, Casellas M, Gaschet M, Dagot C, Ploy MC. Quantitative and qualitative impact of hospital effluent on dissemination of the integron pool. ISME J. 2014;8:768-77.

9. Rodriguez-Mozaz S, Chamorro S, Marti E, Huerta B, Gros M, Sanchez-Melsio A, Borrego CM, Barcelo D, Balcazar JL. Occurrence of antibiotics and antibiotic resistance genes in hospital and urban wastewaters and their impact on the receiving river. Water Res. 2015;69:234-42.

10. Ju F, Li B, Ma L, Wang Y, Huang D, Zhang T. Antibiotic resistance genes and human bacterial pathogens: Co-occurrence, removal, and enrichment in municipal sewage sludge digesters. Water Res. 2016;91:1-10.

11. Love MI, Huber W, Anders S. Moderated estimation of fold change and dispersion for RNA-seq data with DESeq2. Genome Biol. 2014;15:550.

12. Zhu W, Lomsadze A, Borodovsky M. Ab initio gene identification in metagenomic sequences. Nucleic Acids Res. 2010;38:e132.

13. Hyatt D, Chen GL, Locascio PF, Land ML, Larimer FW, Hauser LJ. Prodigal: prokaryotic gene recognition and translation initiation site identification. BMC Bioinformatics. 2010;11:119.

14. Wood DE, Salzberg SL. Kraken: ultrafast metagenomic sequence classification using exact alignments. Genome Biol. 2014;15:R46.

15. Chen C, Pankow CA, Oh M, Heath LS, Zhang L, Du P, Xia K, Pruden A. Effect of antibiotic use and composting on antibiotic resistance gene abundance and resistome risks of soils receiving manurederived amendments. Environ Int. 2019;128:233-43.

16. Hsu LY, Tan TY, Tam VH, Kwa A, Fisher DA, Koh TH. S. Network for Antimicrobial Resistance, Surveillance and correlation of antibiotic prescription and resistance of Gram-negative bacteria in Singaporean hospitals. Antimicrob Agents Chemother. 2010;54:1173-8.

17. Willmann M, Marschal M, HolzI F, Schroppel K, Autenrieth IB, Peter S. Time series analysis as a tool to predict the impact of antimicrobial restriction in antibiotic stewardship programs using the example of multidrug-resistant Pseudomonas aeruginosa. Antimicrob Agents Chemother. 2013;57:1797-803.

18. Lawes T, Lopez-Lozano JM, Nebot CA, Macartney G, Subbarao-Sharma R, Dare CR, Wares KD, Gould IM. Effects of national antibiotic stewardship and infection control strategies on hospital-associated and community-associated meticillin-resistant Staphylococcus aureus infections across a region of Scotland: a non-linear time-series study. Lancet Infect Dis. 2015;15:1438-49.

19. Lawes T, Lopez-Lozano JM, Nebot CA, Macartney G, Subbarao-Sharma R, Wares KD, Sinclair C, Gould IM. Effect of a national 4C antibiotic stewardship intervention on the clinical and molecular 
epidemiology of Clostridium difficile infections in a region of Scotland: a non-linear time-series analysis. Lancet Infect Dis. 2017;17:194-206.

20. Lawes T, Lopez-Lozano JM, Nebot C, Macartney G, Subbarao-Sharma R, Dare CR, Edwards GF, Gould IM. Turning the tide or riding the waves? Impacts of antibiotic stewardship and infection control on MRSA strain dynamics in a Scottish region over 16 years: non-linear time series analysis. BMJ Open. 2015;5:e006596.

21. Qiao M, Ying GG, Singer AC, Zhu YG. Review of antibiotic resistance in China and its environment. Environ Int. 2018;110:160-72.

22. Ribado JV, Ley C, Haggerty TD, Tkachenko E, Bhatt AS, Parsonnet J. Household triclosan and triclocarban effects on the infant and maternal microbiome. EMBO Mol Med. 2017;9:1732-41.

23. Ben W, Wang J, Cao R, Yang M, Zhang Y, Qiang Z. Distribution of antibiotic resistance in the effluents of ten municipal wastewater treatment plants in China and the effect of treatment processes. Chemosphere. 2017;172:392-8.

24. Zhao R, Feng J, Liu J, Fu W, Li X, Li B. Deciphering of microbial community and antibiotic resistance genes in activated sludge reactors under high selective pressure of different antibiotics. Water Res. 2019;151:388-402.

25. Li B, Yang Y, Ma L, Ju F, Guo F, Tiedje JM, Zhang T. Metagenomic and network analysis reveal wide distribution and co-occurrence of environmental antibiotic resistance genes. ISME J. 2015;9:2490502.

26. Ma L, Li B, Jiang XT, Wang YL, Xia Y, Li AD, Zhang T. Catalogue of antibiotic resistome and hosttracking in drinking water deciphered by a large scale survey. Microbiome. 2017;5:154.

27. Cox G, Wright GD. Intrinsic antibiotic resistance: mechanisms, origins, challenges and solutions. Int J Med Microbiol. 2013;303:287-92.

28. Martinez JL, Baquero F, Andersson DI. Predicting antibiotic resistance. Nat Rev Microbiol. 2007;5:958-65.

29. Hu Y, Gao GF, Zhu B. The antibiotic resistome: gene flow in environments, animals and human beings. Front Med. 2017;11:161-8.

30. Arepyeva MA, Kolbin AS, Sidorenko SV, Lawson R, Kurylev AA, Balykina YE, Mukhina NV, Spiridonova AA. A mathematical model for predicting the development of bacterial resistance based on the relationship between the level of antimicrobial resistance and the volume of antibiotic consumption. J Glob Antimicrob Resist. 2017;8:148-56.

31. Turnidge J, Christiansen K. Antibiotic use and resistance-proving the obvious. Lancet. 2005;365:548-9.

32. Selvam A, Xu D, Zhao Z, Wong JW. Fate of tetracycline, sulfonamide and fluoroquinolone resistance genes and the changes in bacterial diversity during composting of swine manure. Bioresour Technol. 2012;126:383-90.

33. Johnsen PJ, Townsend JP, Bohn T, Simonsen GS, Sundsfjord A, Nielsen KM. Factors affecting the reversal of antimicrobial-drug resistance. Lancet Infect Dis. 2009;9:357-64. 
34. Canton R, Ruiz-Garbajosa P. Co-resistance: an opportunity for the bacteria and resistance genes. Curr Opin Pharmacol. 2011;11:477-85.

35. Tacao M, Moura A, Correia A, Henriques I. Co-resistance to different classes of antibiotics among ESBL-producers from aquatic systems. Water Res. 2014;48:100-7.

36. Baker-Austin C, Wright MS, Stepanauskas R, McArthur JV. Co-selection of antibiotic and metal resistance. Trends Microbiol. 2006;14:176-82.

37. Martins ER, Casella T, Bueno MFC, Francisco GR, Tolentino FM, de Freitas ACT, Cerdeira L, Costa MN, Cevada C, Lincopan N, Garcia DO, Nogueira MCL. Draft genome sequence of an aminoglycosideresistant RmtD2-producing Enterobacter cloacae subsp. cloacae ST395 in Brazil. J Glob Antimicrob Resist. 2017;10:308-9.

38. Di Cesare A, Eckert EM, D'Urso S, Bertoni R, Gillan DC, Wattiez R, Corno G. Co-occurrence of integrase 1 , antibiotic and heavy metal resistance genes in municipal wastewater treatment plants. Water Res. 2016;94:208-14.

39. Gillings MR, Gaze WH, Pruden A, Smalla K, Tiedje JM, Zhu YG. Using the class 1 integron-integrase gene as a proxy for anthropogenic pollution. ISME J. 2015;9:1269-79.

40. Chen Z, Zhang W, Yang L, Stedtfeld RD, Peng A, Gu C, Boyd SA, Li H. Antibiotic resistance genes and bacterial communities in cornfield and pasture soils receiving swine and dairy manures. Environ Pollut. 2019;248:947-57.

41. Dahmen S, Poirel L, Mansour W, Bouallegue O, Nordmann P. Prevalence of plasmid-mediated quinolone resistance determinants in Enterobacteriaceae from Tunisia. Clin Microbiol Infect. 2010;16:1019-23.

42. Chouchani C, Marrakchi R, El A, Salabi. Evolution of beta-lactams resistance in Gram-negative bacteria in Tunisia. Crit Rev Microbiol. 2011;37:167-77.

43. Munck C, Albertsen M, Telke A, Ellabaan M, Nielsen PH, Sommer MOA. Limited dissemination of the wastewater treatment plant core resistome. Nat Commun. 2015;6:8452.

44. Chagas TP, Seki LM, Cury JC, Oliveira JA, Davila AM, Silva DM, Asensi MD. Multiresistance, betalactamase-encoding genes and bacterial diversity in hospital wastewater in Rio de Janeiro, Brazil. J Appl Microbiol. 2011;111:572-81.

45. Li J, Cheng W, Xu L, Strong PJ, Chen H. Antibiotic-resistant genes and antibiotic-resistant bacteria in the effluent of urban residential areas, hospitals, and a municipal wastewater treatment plant system. Environ Sci Pollut Res Int. 2015;22:4587-96.

46. Hocquet D, Muller A, Bertrand X. What happens in hospitals does not stay in hospitals: antibioticresistant bacteria in hospital wastewater systems. J Hosp Infect. 2016;93:395-402.

47. Berendonk TU, Manaia CM, Merlin C, Fatta-Kassinos D, Cytryn E, Walsh F, Burgmann H, Sorum H, Norstrom M, Pons MN, Kreuzinger N, Huovinen P, Stefani S, Schwartz T, Kisand V, Baquero F, Martinez JL. Tackling antibiotic resistance: the environmental framework. Nat Rev Microbiol. 2015;13:310-7.

\section{Figures}




\section{Antibiotic dosage}

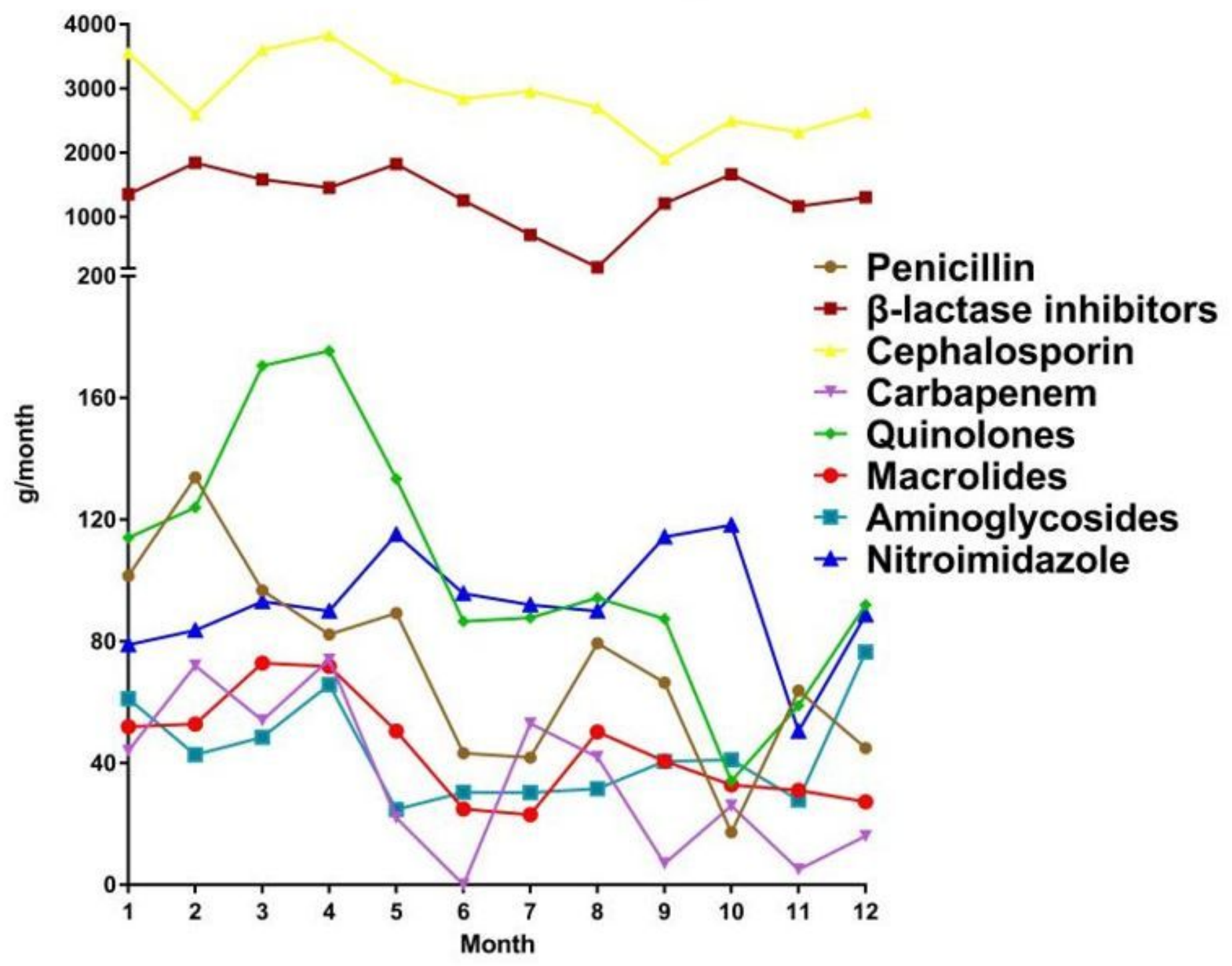

Figure 1

Clinical antibiotic usage in 2018 


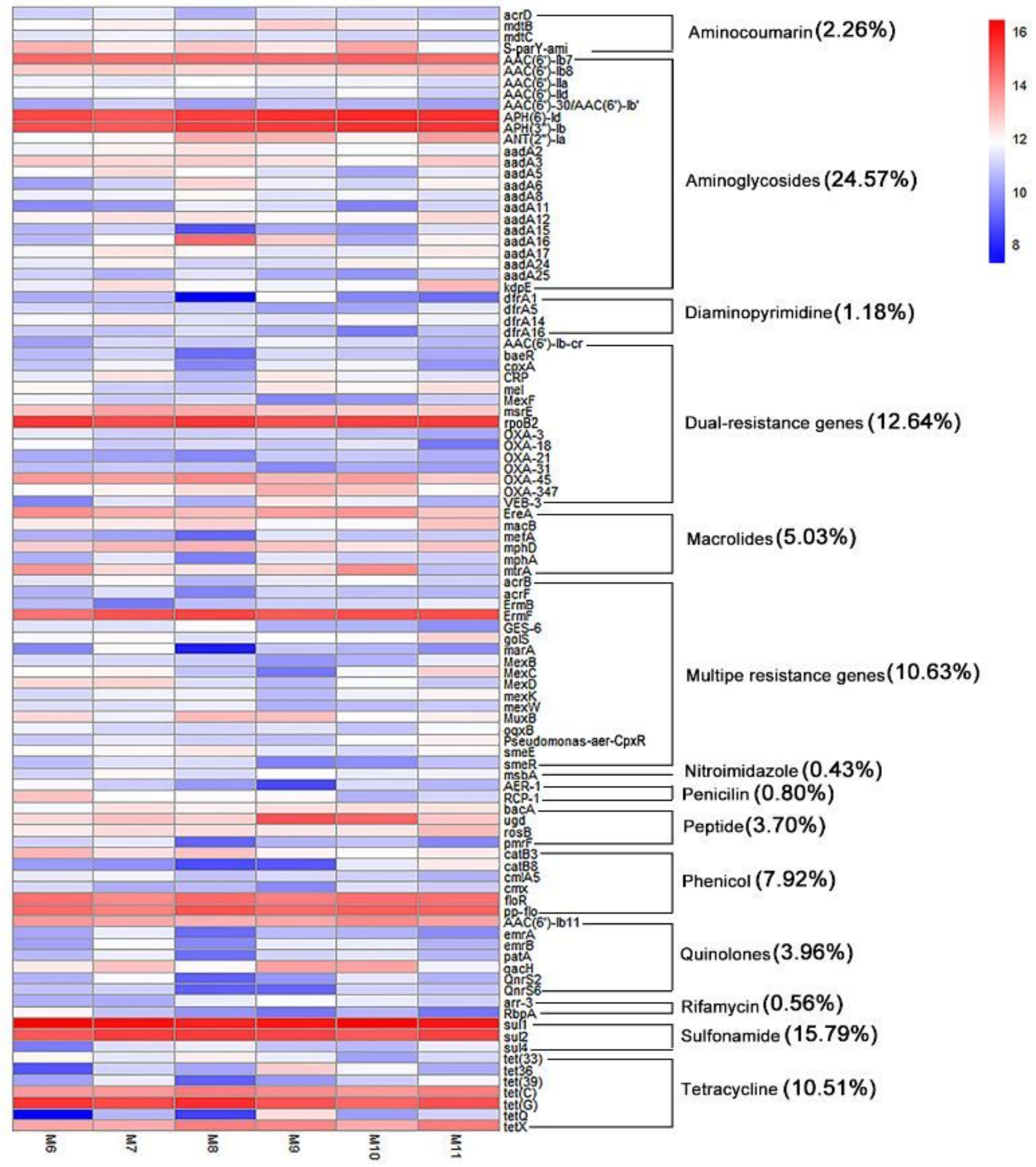

Figure 2

Heat map of resistance genes (abundance of the first 100 resistance genes) 

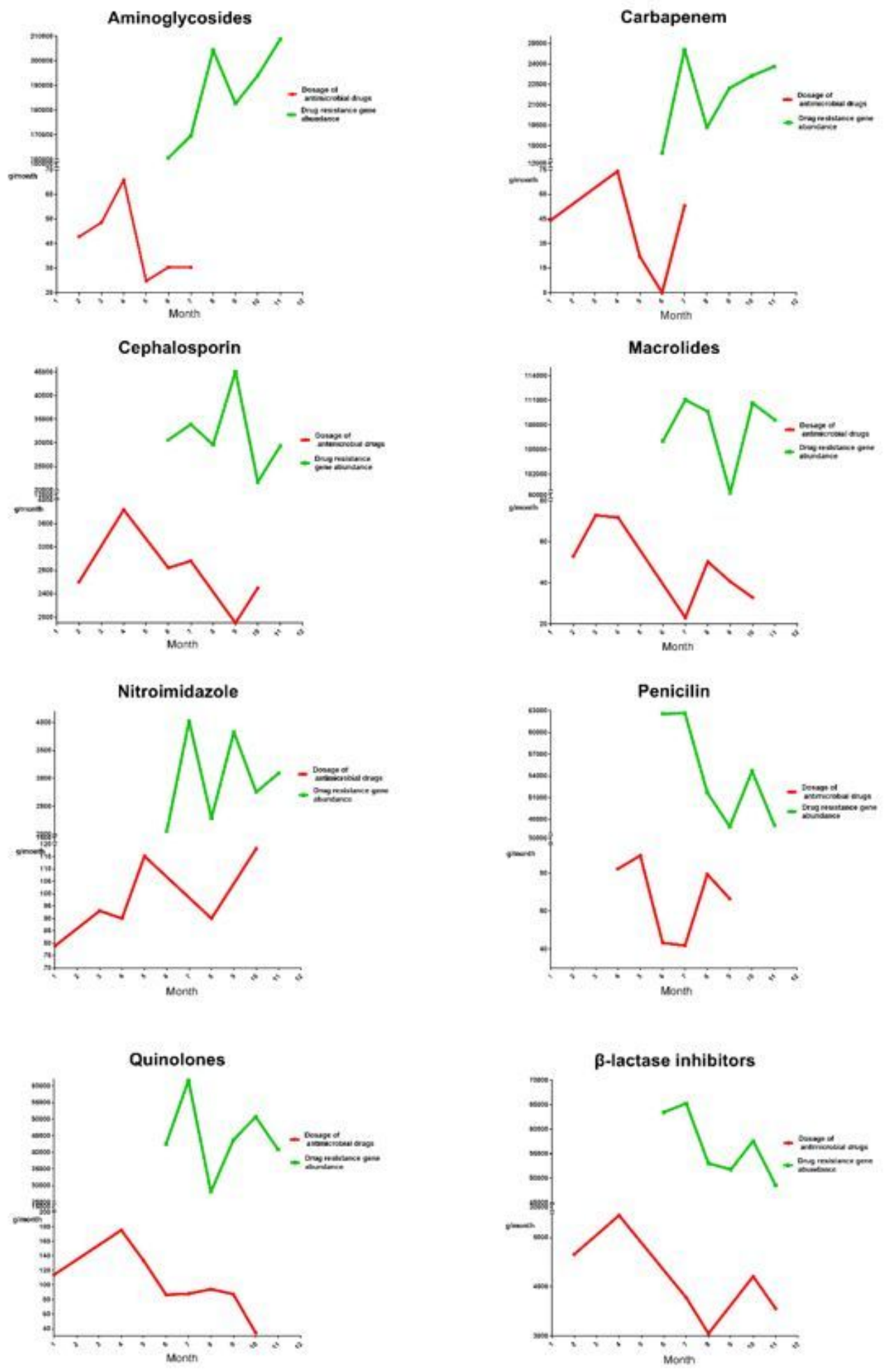

\section{Figure 3}

Relationship between the abundance of ARGs and the corresponding antibiotic 


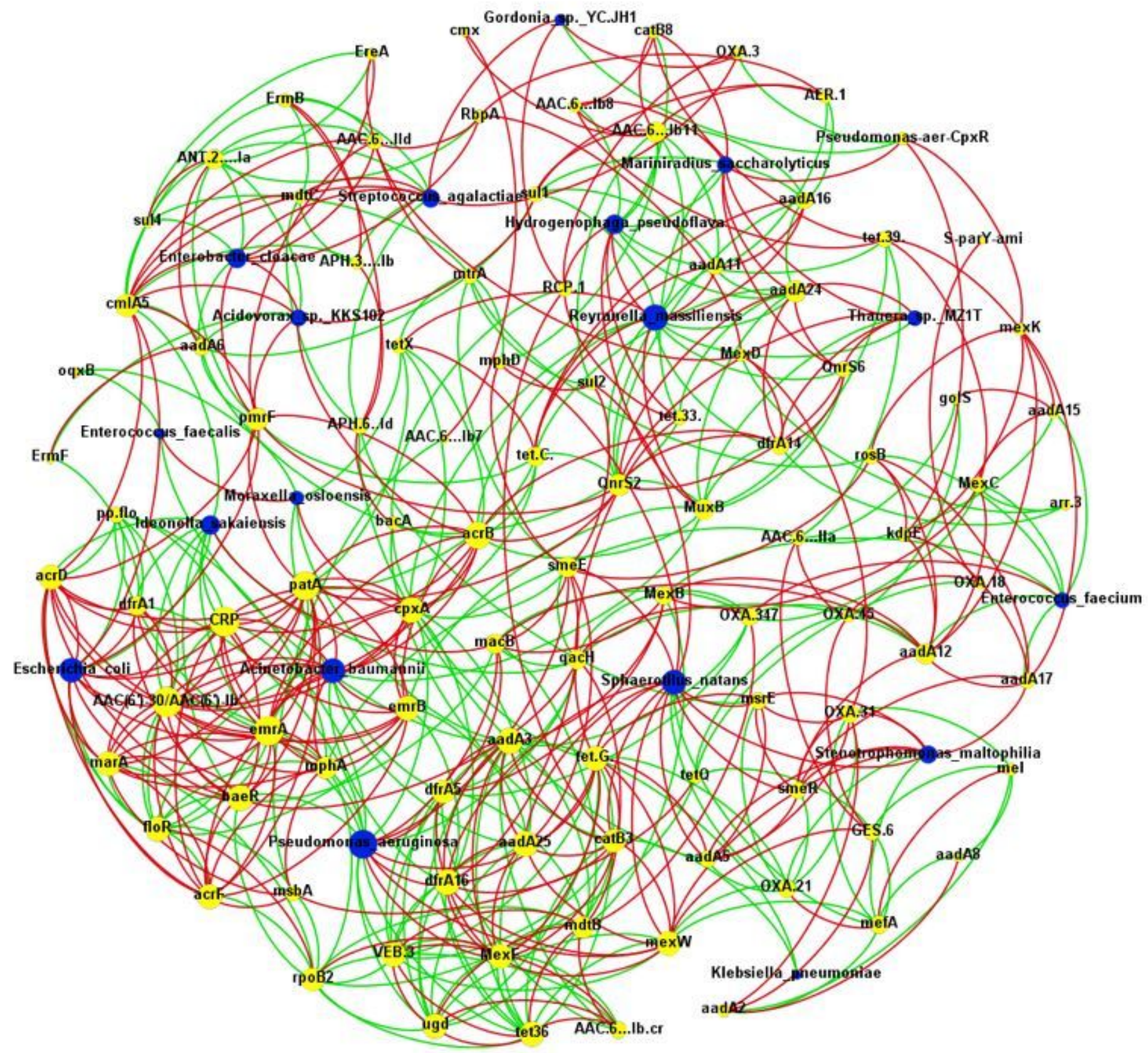

Figure 4

Network co-occurrence pattern of drug-resistant bacteria (ARB) and resistance genes (ARGs) Note: Yellow node represents ARG, blue node represents bacterial species, red lines represent positive correlations, and green lines represent negative correlations. The larger the node, the more connection lines. 


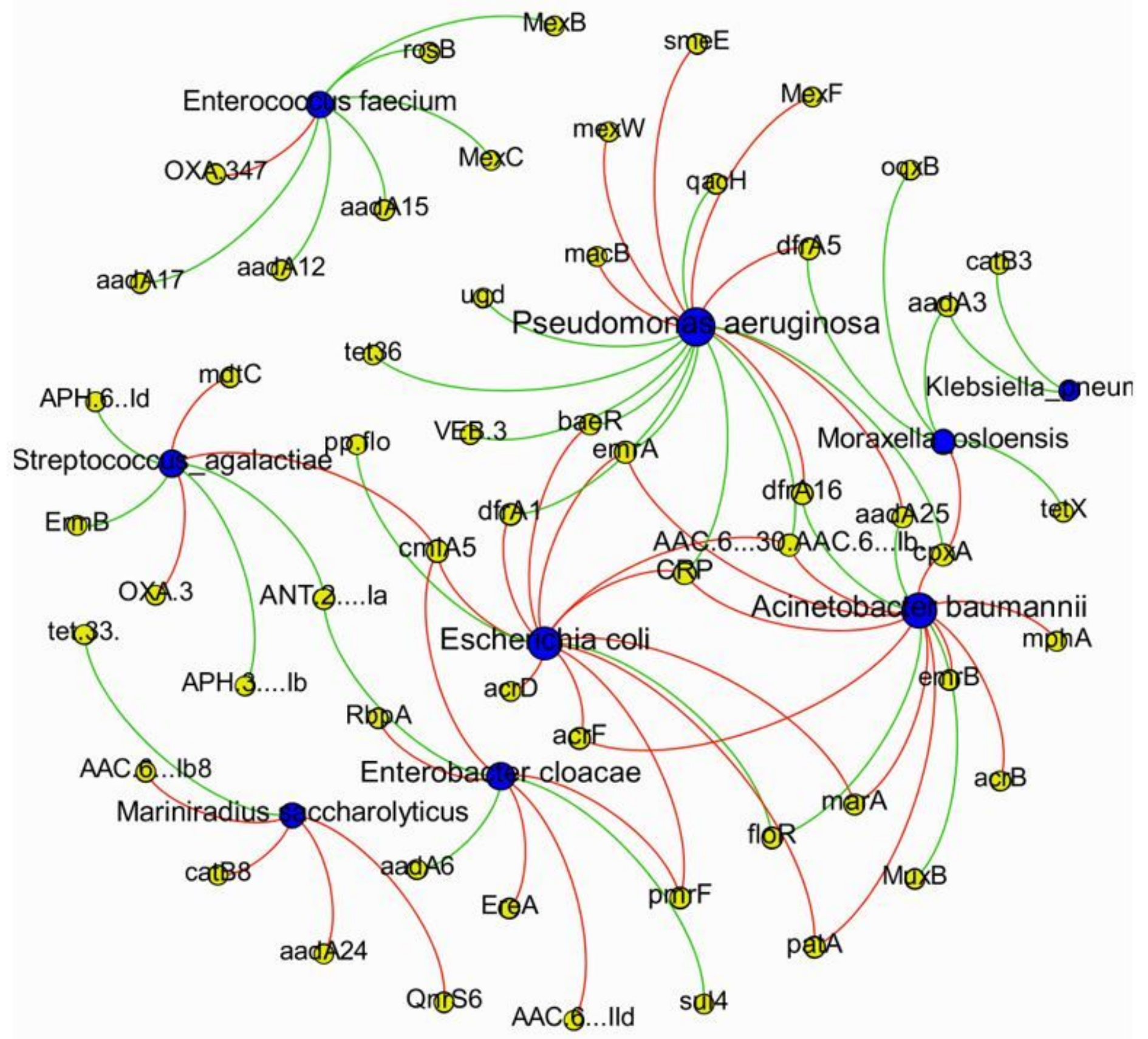

Figure 5

Network co-occurrence model of clinically common bacteria and resistance genes in sewage E. coli was correlated with $A A C\left(6^{\prime}\right)-30 / A A C\left(6^{\prime}\right) \mid b^{\prime}$, acrD, acrF, baeR, CRP, cmlA5, emrA, marA, patA, pmrF, floR, and pp.flo. P. aeruginosa was correlated with aadA25, dfrA16, dfrA5, macB, MexF, mexW, smeE, AAC.6...30/AAC.6...Ib., baeR, cpxA, CRP, dfrA1, emrA, qacH, tet36, ugd, and VEB. A._baumannii correlated with AAC.6...30/AAC.6...Ib., aadA25, acrB, acrF, cpxA, CRP, dfrA16, emrA, emrB, floR, marA, mphA, MuxB, and patA.

\section{Supplementary Files}


This is a list of supplementary files associated with this preprint. Click to download.

- SupplementaryMaterial.docx 